\title{
Endoscopic Management of ERCP Bleeding
}

Fawzy M. Mustafa ${ }^{(1)}$ and Hany F. Ali ${ }^{(2)}$

Department of General Surgery ${ }^{(1)}$ and Clinical Pathology ${ }^{(2)}$, Faculty of Medicine, Al-Azhar University, Cairo, Egypt

\begin{abstract}
Background: post endoscopic retrograde cholangiopancreatography (ERCP) bleeding is a common complication and the incidence varies from $1 \%$ to $48 \%$. It can be challenging to manage or localize the bleeder through a sideviewing endoscope. Objective: this study aimed to evaluate the risk factors of post ERCP bleeding and efficacy of endoscopic intervention therapies.

Patients and Methods: this prospective randomized clinical trial was carried out on 100 consecutive patients at General Surgery Department Al-Azhar University Hospitals (Cairo) Egypt. All patients underwent ERCP, between January 2016 and August 2018, All ES procedures were performed by experienced surgeons and the decision to perform sphincterotomy was based on clinical, endoscopic, and radiologic findings.

Results: comparison of different therapeutic modalities showed that cholangitis was higher in patients who received epinephrine spray and pancreatitis was higher in patients who received electrocoagulation.

Conclusion: clinically, endoscopic hemostasis therapy is very effective to manage post ERCP bleeding but increases the complication rate of pancreatitis and cholangitis. Realizing the effectiveness of each therapeutic modalities and appropriate management of different levels of bleeding are important.
\end{abstract}

Keywords: ERCP

\section{INTRODUCTION}

Endoscopic retrograde cholangiopancreatography (ERCP) is a fundamental tool for the evaluation and management of many pancreaticobiliary diseases ${ }^{(\mathbf{1})}$.

The development of safer and relatively noninvasive investigations such as magnetic resonance cholangiopancreatography and endoscopic ultrasound has meant that ERCP is now rarely performed without therapeutic intent ${ }^{(2)}$.

The major risk of an ERCP is the development of pancreatitis, intestinal perforation and sphincterotomy is also associated with a risk of bleeding ${ }^{(3)}$.

Bleeding is one of the serious complications of therapeutic ERCP and can be mostly attributed to endoscopic sphincterotomy (ES), which is a fundamental step of the procedure (ERCP) for accessing the biliary and pancreatic ductal systems ${ }^{(4)}$.

The timing of delayed post-ES bleeding may be immediate or up to 10 days following ES. A small amount of post-ES bleeding is common and most often resolves spontaneously. Thus, endoscopic therapy was suggested to be undertaken for the treatment of endoscopically significant immediate bleeding or clinically significant delayed bleeding. However, some cases present with significant bleeding that requires blood transfusions and urgent endoscopic intervention, such as injection, balloon tamponade, thermal, and mechanical methods (hemoclip or self-expandable stent) alone or in combination. If refractory bleeding occurs, repeated endoscopic hemostatic therapy, angiographic embolization, or surgery is required ${ }^{(5)}$.

Owing to the tangential approach in these patients, the bleeding could not be handled easily with side-viewing endoscope and this urgent procedure could not be practically performed by the gastroenterologists experienced in ERCP each time. Furthermore, bleeding that obscures the visual field makes identification of the precise location in delayed bleeding impossible ${ }^{(5)}$.

The complications of pancreatitis, cholangitis, and cholecystitis associated with endoscopic hemostasis were higher in patients who underwent endoscopic hemostasis than in patients who did not undergo endoscopic hemostasis ${ }^{(\mathbf{6})}$.

Post-ERCP bleeding is a serious complication that can lead to morbidity and mortality. Effective management and prevention of this complication requires knowledge of its risk factors and proficiency in endoscopic hemostasis techniques through asideviewing duodenoscope ${ }^{(7)}$.

\section{AIM OF THE STUDY}

The aim of the paper is to establish a wellaccepted clinical strategy for management of post ERCP bleeding in patients with or without coagulopathy defects.

\section{PATIENTS AND METHODS}

This prospective randomized clinical trial was carried out on 100 consecutive patients at General Surgery Department Al-Azhar University Hospitals, Cairo, Egypt.

All patients underwent ERCP, between January 2016 and August 2018. All ES procedures were performed by experienced surgeons and the decision to perform sphincterotomy was based on clinical, endoscopic, and radiologic findings.

\section{Written informed consent:}

An approval of the study was obtained from Al- Azhar University academic and ethical 
committee. Every patient signed an informed written consent for acceptance of the operation.

The ERCP procedure was carried out either under conscious sedation with midazolam (2-10 mg intravenously [IV]) and fentanyl (0.05-0.1 mg IV) or with the patient under general anesthesia. In addition, intravenous hyoscine butylbromide (40-60 $\mathrm{mg}$ IV) or glucagon (1-2 mg IV) was administered.

All patients were subjected to: Clinical evaluation including: history taking, general examination, abdominal examinations, Laboratory investigations (complete blood count, prothrombin time and activity and INR, liver and kidney functions, fasting and two hours postprandial blood glucose level, serum electrolytes: (sodium, potassium, total and ionized calcium) and hepatitis C and B markers) and Imaging evaluation included transabdominal ultrasonography and chest $\mathrm{x}$ ray, computerized tomography and MRCP if indicated, ECG, echocardiography and pulmonary function tests.

Clinical and laboratory parameters before and after ERCP were analyzed to identify the risk factors and outcome of bleeding as a complication of ERCP.

Patients were divided into two groups (Group A 50 patients) with risk of bleeding and coagulopathy defect (thrombocytopenia or coagulation factor defects) 1-Thrombocytopenia was defined as a thrombocyte count of less than 150,000/uL. 2International normalized ratio (INR) greater than 1.2 was defined as prolonged. INR levels between 1.2 and 1.5 were defined as impaired. INR was defined as corrected if levels were decreased to less than 1.5 after treatment with fresh frozen plasma (FFP) and/or vitamin K. 3-Anticoagulant therapy within 3 days. 4-Liver cirrhosis 5- Renal impairment 6- The use of NSAIDs and (Group B 50 patients) with no risk of bleeding and without coagulopathy defect.

\section{Inclusion criteria:}

All patients were presented with uncontrollable bleeding during or after an ERCP procedure (up to 15 days afterward) were included prospectively.

When bleeding occurred during ERCP, it was considered uncontrollable if hemorrhage was so massive in amount (oozing or arterial type) that it prevented further biliary interventions, this is because there was no clear visualization of the area and / or if it continued despite conventional endoscopic hemostatic treatment, for at least 5 minutes.

If post-ES bleeding occurred, endoscopic therapy was performed using a side-viewing or end-viewing endoscope, with the type depending on the endoscopist's discretion and one or more of the following methods was done:

1-Epinephrine was either injected $(1: 10,000$ dilutions; $3 \pm 20 \mathrm{~mL}$ ) or irrigated (1:50,000 dilutions; $30 \pm 50 \mathrm{~mL}$ )

2-Thermotherapy was attempted with the sphincterotome wire or heater probe, and the power setting was on forced coagulation mode of $30 \mathrm{~W}$.

3-Balloon tamponade was performed with a dilating catheter $(10 \mathrm{~mm} \times 4 \mathrm{~cm})$, and ballooning time was 3 -5 minutes and can be repeated.

4-Hemostasis could be achieved by precise placement of one or more hemoclips at the bleeding site (if bleeder identified). 5-Self-expandable biliary stent

Severity of bleeding was classified as mild (with endoscopic evidence of bleeding, with a hemoglobin decrease $<3 \mathrm{~g} / \mathrm{dL}$, without any need for blood transfusion), moderate (requires blood transfusion of 4 units or less, without any need for angiographic or surgical intervention), or severe (requires $>4$ units of blood transfusion or intervention)

Onset of bleeding was classified as immediate (hemorrhage at the time of sphincterotomy) or delayed (hemorrhage after sphincterotomy and manifested by melena, hematemesis, or hematochezia associated with a decrease in hemoglobin level).

Endoscopic treatment was considered successful if there was no further bleeding or if recurrent hemorrhage was controlled endoscopically. Two treatment sessions were permitted before endoscopic therapy was considered to have failed.

\section{Follow-up}

At the time of discharge from ERCP unit, patients were specifically instructed not to consume NSAIDs, antiplatelet agents, and coumadin for at least 3 days. In patients with a prolonged INR, FFP infusions were given for 4 to 5 days in order to maintain INR below 1.5. Thrombocyte suspensions were transfused to patients with a thrombocyte count of less than $50,000 / \mathrm{ml}$. 

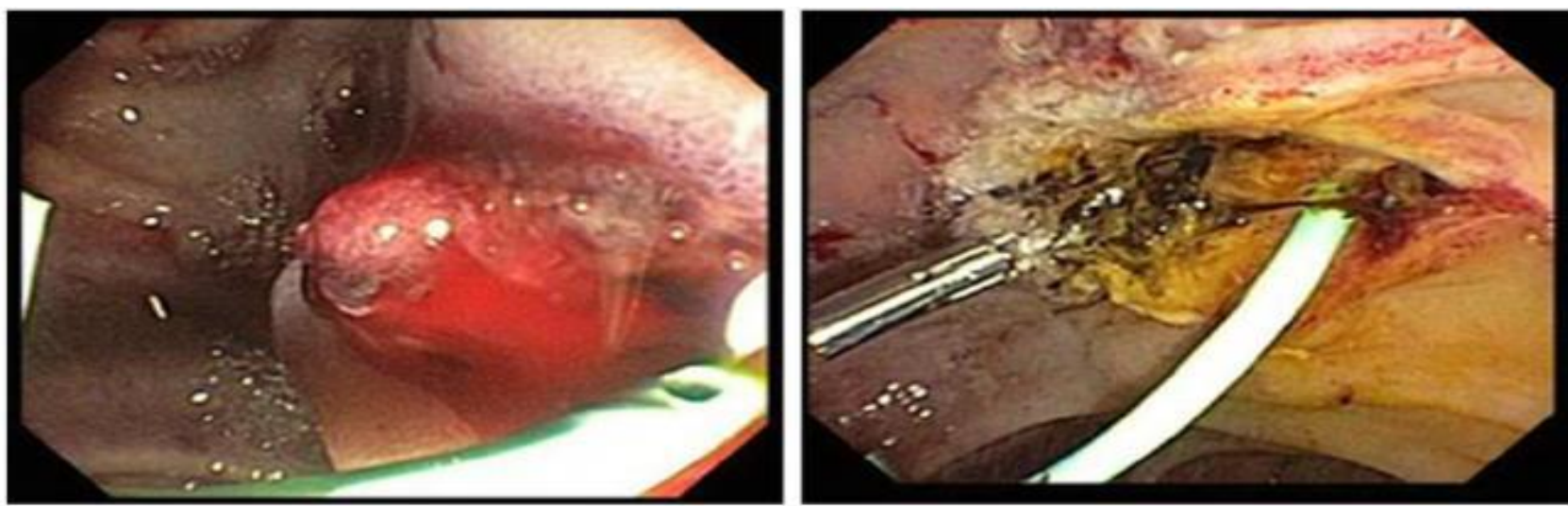

Figure (1): Post-ES bleeding treated with thermal therapy and hemoclip.
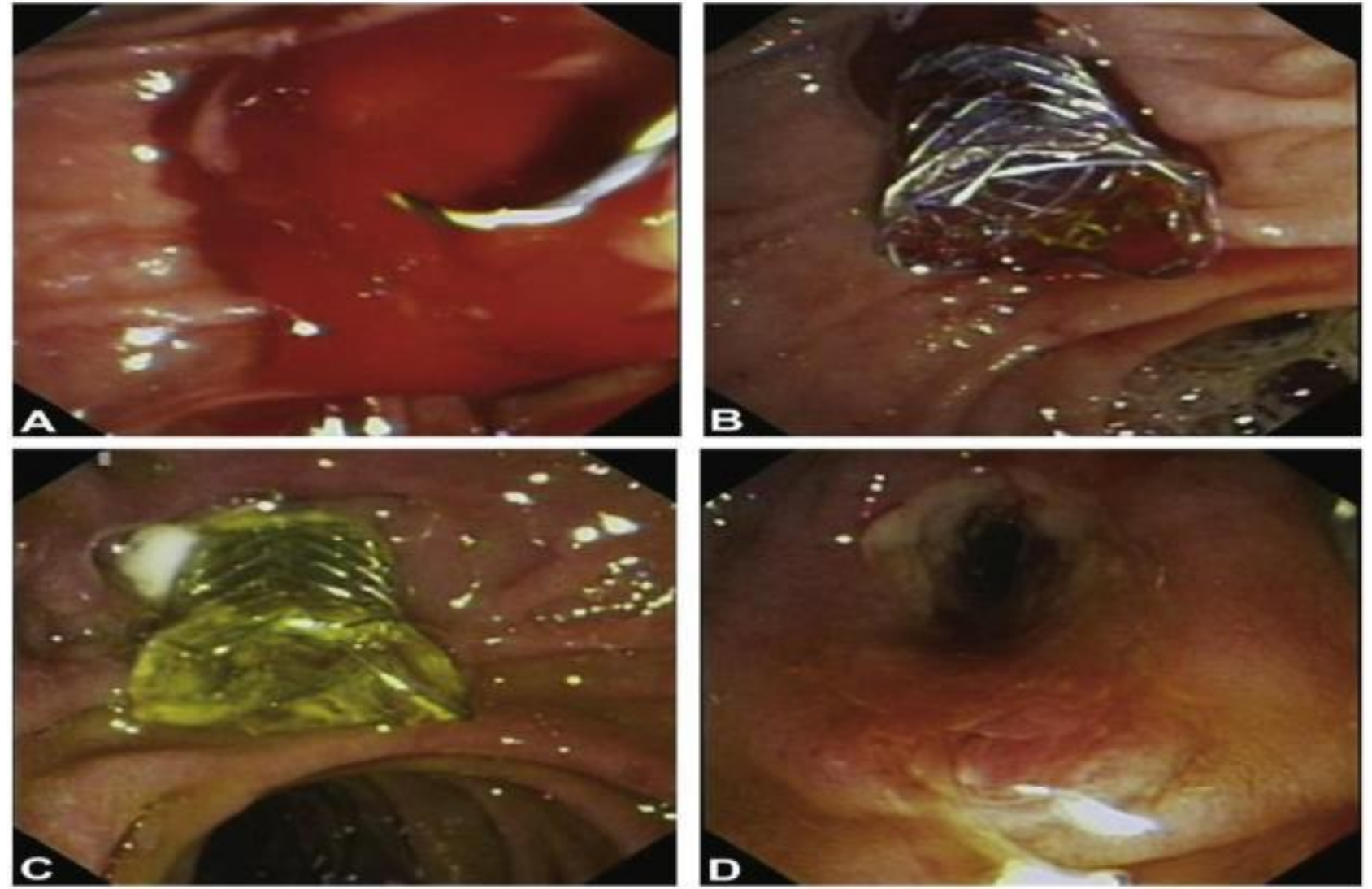

Figure (2): A, Uncontrolled active bleeding. B, Covered metallic biliary stent placement. C, Biliary stent in place 15 days later. D, Papilla after biliary stent removal.

\section{Statistical analysis}

Recorded data were analyzed using the statistical package for social sciences, version 20.0 (SPSS Inc., Chicago, Illinois, USA). Quantitative data were expressed as mean \pm standard deviation (SD). Qualitative data were expressed as frequency and percentage.

\section{The following tests were done:}

- Independent-samples t-test of significance was used when comparing between two means.

- Chi-square $\left(\mathrm{x}^{2}\right)$ test of significance was used in order to compare proportions between two qualitative parameters.

- The confidence interval was set to $95 \%$ and the margin of error accepted was set to $5 \%$. The p-value was considered significant as the following:
- Probability (P-value)

- P-value <0.05 was considered significant.

- P-value <0.001 was considered as highly significant.

- P-value >0.05 was considered insignificant.

\section{RESULTS}

The age of patients ranged between 32 to 57 years with a mean age of $44.5 \pm 12.5 y e a r s$. Sixty three cases were females $(63 / 100,63 \%)$ while thirty-seven cases were males $(37 / 100,37 \%)$ with a female to male ratio of $1.7: 1$ and the statistical analysis revealed that females were more commonly affected than males. Patients were divided into two groups (group A 50 patients and Group B 50 patients) with the following Clinical characteristics. 
Table (1): Clinical characteristics.

\begin{tabular}{|c|c|c|}
\hline Characteristics & Group A & Group B \\
\hline \multicolumn{3}{|l|}{ Reason of ES } \\
\hline CBD stone & 16 & 32 \\
\hline Post biliary complications & 5 & 12 \\
\hline Jaundice due to Pancreatic tumor & 12 & 1 \\
\hline Jaundice due to malignant stricture & 11 & - \\
\hline Sphincter of Oddi dysfunction & 5 & 1 \\
\hline Papillary tumor & 1 & 2 \\
\hline Chronic pancreatitis & - & 2 \\
\hline \multicolumn{3}{|l|}{ Comorbid conditions } \\
\hline Liver cirrhosis & 9 & -- \\
\hline Cardiac disease & 4 & 5 \\
\hline Acute cholangitis & 7 & -- \\
\hline CBD dilation & 14 & 7 \\
\hline Duodenal ulcer & 5 & 3 \\
\hline Renal impairment & 1 & -- \\
\hline Diabetes mellitus & 4 & 5 \\
\hline \multicolumn{3}{|l|}{ Laboratory parameters } \\
\hline Total bilirubin (mg/dL) & $5.86 \pm 7.28$ & $0.77 \pm 4.23$ \\
\hline PT INR & $1.10 \pm 0.25$ & $1.08 \pm 0.10$ \\
\hline Platelet(10^3/uL) & $170.6 \pm 79.9$ & $228.8 \pm 91.0$ \\
\hline Creatinine (mg/dL) & $1.40 \pm 1.72$ & $1.02 \pm 0.76$ \\
\hline
\end{tabular}

Among both groups' variables associated risk factors of post-ES bleeding in the multivariable analysis are presented in group A. Thrombocytopenia was an important risk factor of relapse, the other parameters, liver cirrhosis, end stage renal disease, and anticoagulant therapy within 3 days, remained statistically significant.

Among 100 patients with ERCP, post ESbleeding was recorded in 33 patients (immediate and delayed bleeding were noted in $24(24 \% \%)$ and $9(9$ $\% \%$ ) patients, respectively) all patients were among group A. All immediate bleeding patients received proton pump inhibitor to prevent delayed bleeding.
Five patients (5\%) had rebleeding after initial endoscopic hemostasis.

Delayed bleeding occurred $1 \pm 7$ days (mean, 2.5 days) following the procedures.

Among the 9 patients with delayed bleeding, 7 patients received endoscopic intervention. Five patients received a side-viewing and two patients received end viewing endoscope. Hemostatic therapy was done in seven patients with no failure to achieve initial hemostasis. No patient required angiography or surgery for delayed bleeding. Outcomes of different endoscopic hemostatic therapies are listed in the following Table 2.

Table 2: Outcomes of different endoscopic hemostatic therapies

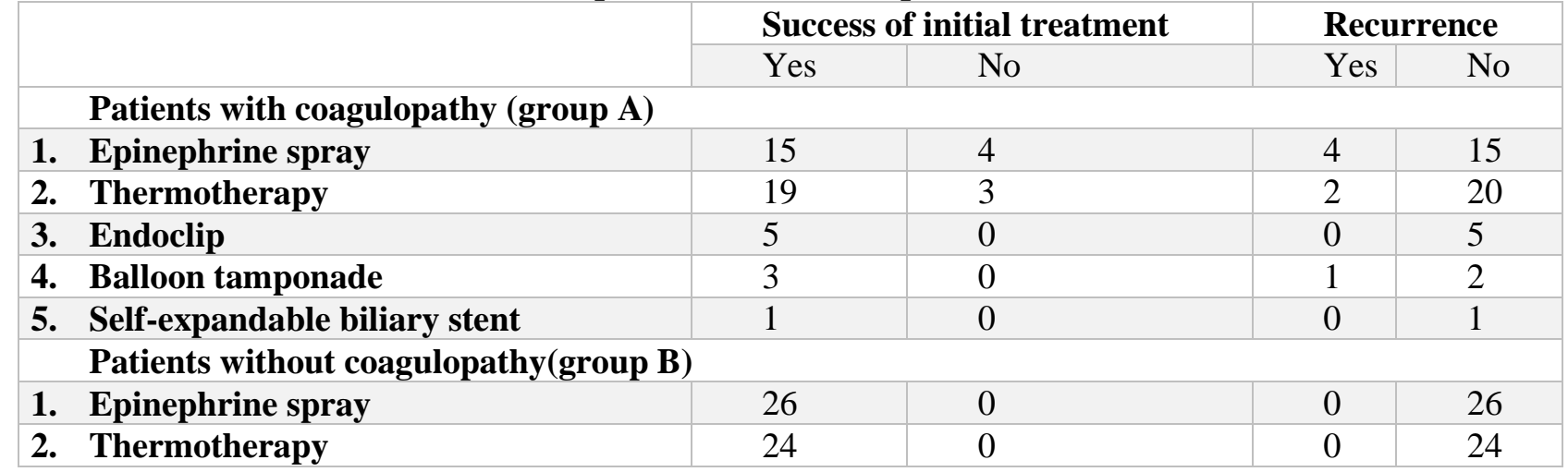


The use of epinephrine spray was associated with higher complication of cholangitis, whereas thermocoagulation and epinephrine injection were associated with higher complication of pancreatitis as shown in the following table 3:

Table 3:

\begin{tabular}{|l|l|l|}
\hline Characteristics & Pancreatitis & Cholangitis \\
\hline Epinephrine spray & 2 & 5 \\
\hline Thermotherapy & 4 & 2 \\
\hline Endoclip & 0 & 0 \\
\hline Balloon tamponade & 1 & 3 \\
\hline $\begin{array}{l}\text { Self-expandable } \\
\text { biliary stent }\end{array}$ & 1 & 2 \\
\hline
\end{tabular}

Post-ES bleeding and endoscopic hemostasis would increase the complication of pancreatitis and cholangitis; but realizing the effectiveness of each therapeutic modality and appropriate management of various degrees of bleeding are important so we should assess benefit risk ratio.

\section{DISCUSSION}

For clinically significant hemorrhage, definite risk factors include any degree of bleeding during the procedure, presence of coagulopathy or thrombocytopenia, initiation of anticoagulant therapy within 3 days after ES, liver cirrhosis, dilated common bile duct, periampullary diverticulum, precut sphincterotomy, and relatively low case volume on the part of the endoscopist (one sphincterotomy per week or fewer ${ }^{(8)}$. This study revealed also that coagulopathy is the most important factor determining persistence of bleeding and recurrence after endoscopic treatment. INR defect was the most significant risk factor that can be controlled pre-procedure by good hydration and vitamin $\mathrm{K}$ injection and this decreases risk of bleeding significantly. Other risk factors include liver cirrhosis, platelet count $<150,000 / \mathrm{uL}$, renal impairment, anticoagulant therapy within 3 days. NSAIDs within 3 days of the procedure is considered as a risk factor for bleeding, which is also accepted by Freeman $\boldsymbol{e t}$ al. ${ }^{(9)}$.

Recently, the European consensus recommended the discontinuation of clopidogrel 5 days before the procedure and continuation of aspirin use for high-risk endoscopic procedures such as ES ${ }^{(\mathbf{1 0})}$. However, an Asian study revealed an increased risk of post-ES bleeding even when aspirin was withheld for 1 week ( $10 \%$ vs. $4 \%, \mathrm{P}=0.03)$, which supports our study to include aspirin use as a risk factor for bleeding ${ }^{(11,12)}$.

Most of the factors predicting the persistence or recurrence of bleeding after endoscopic treatment are similar. A platelet count greater than 50,000 IU/L, and an INR less than 1.5 are usually considered adequate to perform ES. ES was performed after correcting the INR and thrombocyte counts to above defined criteria, and supportive treatment was continued for 3 days after the procedure. Our results highlight the importance of coagulopathy in the development of persistent and recurrent bleeding.

Thus, endoscopic therapy was suggested to be undertaken for the treatment of endoscopically significant immediate bleeding or clinically significant delayed bleeding. However, some cases present with significant bleeding that requires blood transfusions and urgent endoscopic intervention, such as injection, balloon tamponade, thermal, and mechanical methods alone or in combination ${ }^{(\mathbf{1 3})}$.

If refractory bleeding occurs, repeated endoscopic hemostatic therapy, angiographic embolization, or surgery is required ${ }^{\text {(14) }}$.

The post-ES bleeding rate was $33 \%$ in our study, which was higher than those seen in previous studies. The difference may result from dividing patients into two groups with 50 patients with high risk of bleeding including any degree of bleeding during the procedure, presence of coagulopathy or thrombocytopenia, initiation of anticoagulant therapy within 3 days of ES, end-stage renal disease and liver cirrhosis. The easiest, cheapest, and most commonly used treatment of bleeding is injection of dilute epinephrine into and around the sphincterotomy site (15). The necessity of application of heat probe exactly to the bleeding point is accepted as a disadvantage of the method by some of the endoscopists. In contrast, epinephrine injection does not require accurate targeting. Injection close to the bleeding point will suffice to control bleeding, resulting in a better endoscopic view for more accurate targeting of the additional contact thermal therapy. Both heat probe and epinephrine injection were very effective in patients' without coagulopathy whereas recurrence may develop in patients with an underlying coagulopathy even if they received combination treatment $(15,16)$.

The initial success rate of our patients treated with epinephrine injection was $78.95 \%$. with recurrence of bleeding in 4 cases (21.05\%). Among thermal methods, multipolar electrocoagulation, argon plasma coagulation, and monopolar electrocoagulation were found to be effective in $19 / 22$ patients $(86.36 \%$ ) with recurrence of bleeding in 2/22 patients $(9.09 \%$ ).

Balloon tamponade using an ERCP occlusion or dilating balloon might be a useful adjunct tool to manage post-ES bleeding as it often allows temporary control of bleeding and an improvement of visualization for definitive therapy. The insertion of a temporary fully covered self-expandable (SEMS) to achieve immediate hemostasis has been shown to be effective in few cases ${ }^{(\mathbf{1 7})}$.

This form of mechanical tamponade can be considered in patients with difficult to treat post-ES bleeding and when bleeding is originating from deep within the papilla or distal CBD ${ }^{(18)}$.

Stents can be safely removed in 1-2 weeks after placement, and patients on anticoagulant therapy can resume therapy while the stent is in place ${ }^{(16,17)}$. 
In our study, self-expandable biliary stent was done for a refractory case (one case) after failure of epinephrine injection and thermotherapy with excellent control of bleeding and no recurrence while balloon tamponade was used to temporary control 5 cases with good initial hemostasis in four cases and one case needed application of hemoclip with good result. Also Endoclip was an effective treatment in 5 cases (identified bleeder) with no recurrence after initial control of bleeding so we recommend this method if identified bleeder was detected.

We agree that in delayed bleeding cases, repeat the endoscopic procedure (epinephrine injection and / or thermal coagulation) or combine endoscopic therapies to achieve hemostasis. But this strategy failed to control bleeding in few cases that need other methods to control bleeding such as clip, covered selfexpandable metal stent application, and balloon tamponade ${ }^{(\mathbf{1 9})}$.

Fully covered metallic biliary stents have also been applied in post-ES bleeding and have afforded good outcomes. However; higher cost effect limits its use to refractory cases ${ }^{(20)}$. Complications associated with endoscopic hemostasis may occur, but it may be difficult to distinguish which of these were related to the ERCP itself and which were related to the treatment of bleeding ${ }^{(7)}$. Outcomes of different endoscopic hemostatic therapies in this study revealed that the use of epinephrine spray was associated with higher complication of cholangitis, whereas thermocoagulation and epinephrine injection were associated with higher complication of pancreatitis. The outcome of the endoclip was not statistically different.

\section{CONCLUSION}

1. Mild post-ES bleeding could be managed with proton pump inhibitors because this drug could promote thrombus formation and stop bleeding from small vessels.

2. As for larger blood vessel damage or arterial hemorrhage, pharmacological treatment combined with endoscopic treatment is necessary.

3. Endoscopic interventional therapy for immediate post-ES bleeding may lead to better localization of bleeding point and prevention of delayed bleeding.

4. The patients with delayed bleeding are better to receive endoscopic evaluation, and good hemostasis.

Therefore, suitable knowledge of management of different severities of bleeding and complication of therapy are important.

\section{REFERENCES}

1. Arvanitakis M, Deviere J (2009): Endoscopic retrograde cholangio pancreatography (ERCP). Endoscopy, 41:890-4.

2. McAlister VC, Davenport E, Renouf E et al. (2007): Cholecystectomy deferral in patients with endoscopic sphincterotomy. Cochrane Database Syst Rev., CD006233.

3. Andriulli A, Loperfido S, Napolitano G et al. (2007): Incidence rates of post-ERCP complications: a systematic survey of prospective studies. Am J Gastroenterol., 102(8): 1781-8.

4. Kang MH, Jeong JW and Han JH (2014): Successful hemostasis by using endoscopic band ligation for uncontrolled postendoscopic sphincterotomy bleeding. Gastrointest Endosc., 79:336-339.

5. Ferreira LE, Baron TH (2007): Post-sphincterotomy bleeding: who, what, when, and how. AmJ Gastroenterol., 102:2850-8.

6. Christensen M, Matzen P, Schulze S et al. (2004): Complications of ERCP: a prospective study. Gastrointest Endosc., 60:721-726.

7. Hassan M, Barham K (2014): Hemorrhagic complications following endoscopic retrograde cholangiopancreatography . Techniques in Gastrointestinal Endoscopy, 16(2014) 175182.

8. Hori Y, Naitoh I, Nakazawa T et al. (2014): Feasibility of endoscopic retrograde cholangiopancreatography-related procedures in hemodialysis patients. J Gastroenterol Hepatol., 29:648.

9. Freeman ML, Nelson DB, Sherman S et al. (1996): Complications of endoscopic biliary sphincterotomy. N Engl J Med., 335:909-18.

10. Shomei R, Naoki U, Hirotoshi I et al. (2007): Management of endoscopic sphincterotomy-induced bleeding. Digestive Endoscopy, 19(1): 60-63.

11. Wang Y, Han Z, Niu X et al. (2015): Clinical research for delayed hemorrhage after endoscopic sphincterotomy. Int J Clin Exp Med., 8(4): 5753-9.

12. Hui CK, Lai KC, Yuen MF et al. (2002): Does withholding aspirin for one week reduce the risk of post sphincterotomy bleeding? Aliment Pharmacol Ther., 16(5): 929-36.

13. Wilcox CM, Canakis J, Monkemuller KE et al. (2004): Patterns of bleeding after endoscopic sphincterotomy, the subsequent risk of bleeding, and the role of epinephrine injection. AmJ Gastroenterol., 99:244-8.

14. Saeed M, Kadir S, Kaufman SL et al. (1989): Bleeding following endoscopic sphincterotomy: Angiographic management by transcatheter embolization. Gastrointest Endosc., 35(4): 300-3.

15. Verma D, Kapadia A and Adler DG (2007): Pure versus mixed electrosurgical current for endoscopic biliary sphincterotomy: a meta-analysis of adverse outcomes. Gastrointest Endosc., 66:283-90.

16. Itoi T, Yasuda I, Doi $\mathrm{S}$ et al. (2011): Endoscopic hemostasis using covered metallic stent placement for uncontrolled post-endoscopic sphincterotomy bleeding. Endoscopy, 43:369-373.

17. Zippi M, DeFelici I, Pica R et al. (2013): Self- expandable metal stent placement for treatment of severe sphincterotomy bleeding. Clin Ter., 164:27-9.

18. Canena J, Liberato M, Horta D et al. (2013): Short-terms tenting using fullycoveredself-expandable metal stents for treatmen to frefractory biliary leaks, post sphincterotomy bleeding, and perforations. Surg Endosc., 27: 313-24.

19. Takahara N, Isayama H, Sasaki T et al. (2012): Endoscopic papillary balloon dilation for bile duct stones in patients on hemodialysis. J Gastroenterol., 47:918-23.

20. Baron TH, Harewood GC (2004). Endoscopic balloon dilation of the biliary sphincter compared to endoscopic biliary sphincterotomy for removal of common bile duct stones during ERCP: a Meta-analysis of randomized, controlled trials. Am J Gastroenterol., 99:1455-60. 